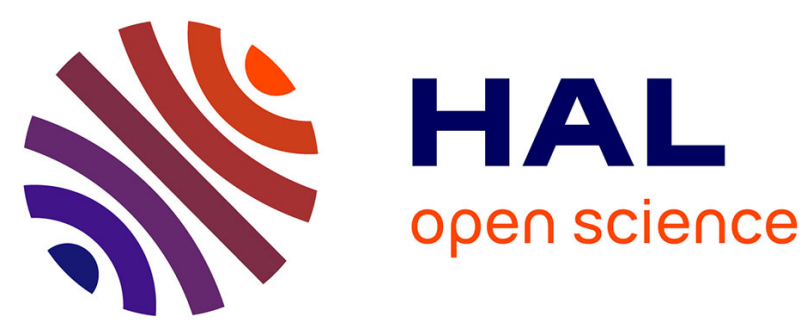

\title{
Simultaneous amplitude and frequency noise analysis in Chua's circuit
}

\author{
Jean-Michel Friedt, Daniel Gillet, Michel Planat
}

\section{To cite this version:}

Jean-Michel Friedt, Daniel Gillet, Michel Planat. Simultaneous amplitude and frequency noise analysis in Chua's circuit. International journal of bifurcation and chaos in applied sciences and engineering, 2003, 13, pp.2301-2308. 10.1142/S0218127403007953 . hal-00078147

\section{HAL Id: hal-00078147 \\ https://hal.science/hal-00078147}

Submitted on 25 May 2021

HAL is a multi-disciplinary open access archive for the deposit and dissemination of scientific research documents, whether they are published or not. The documents may come from teaching and research institutions in France or abroad, or from public or private research centers.
L'archive ouverte pluridisciplinaire $\mathbf{H A L}$, est destinée au dépôt et à la diffusion de documents scientifiques de niveau recherche, publiés ou non, émanant des établissements d'enseignement et de recherche français ou étrangers, des laboratoires publics ou privés. 


\title{
SIMULTANEOUS AMPLITUDE AND FREQUENCY NOISE ANALYSIS IN CHUA'S CIRCUIT
}

\author{
J.-M. FRIEDT \\ IMEC, MCP/BIO, Kapeldreef 75, 3001 Leuven, Belgium \\ D. GILLET and M. PLANAT \\ Laboratoire de Physique et Métrologie des Oscillateurs (LPMO, CNRS) 32, \\ avenue de l'observatoire, 25044 Besançon Cedex, France
}

\begin{abstract}
A large number of simultaneous frequency and amplitude data from an electronic chaotic circuit (Chua's circuit) have been obtained. These acquisitions are validated by plotting the bifurcation diagrams of the experimental data versus the bifurcation parameter. We introduce a topological parallel between the Colpitts oscillator and Chua's circuit, and look for similar behavior of the frequency fluctuations using the Allan deviation.
\end{abstract}

Keywords: Chua's circuit; frequency fluctuations; Colpitts oscillator; amplitude; Allan deviation.

\section{Introduction}

Chua's circuit is a nonlinear electronic circuit which exhibits chaotic behavior for high enough values of one of its parameters, the inductor's value in our case. We have studied the characteristics of the frequency and amplitude fluctuations of the oscillator in the periodic and chaotic regimes (while the signal is still pseudo-periodic, i.e. before the bifurcation towards the double-scroll regime). The amplitude and period of successive oscillations were measured and analyzed by plotting the return maps, the transfer functions and the bifurcation diagrams. The type of fluctuations were characterized using the Allan deviation, and compared to the classical results obtained for a quartz resonator based Colpitts oscillator.

\section{Motivation of This Study}

Our aim is to be able to characterize frequency instabilities in oscillators [Eckert et al., 1996]. We have thus chosen to analyze Chua's chaotic circuit as an example of a highly unstable oscillator. The conclusions can be tentatively extended to more classical oscillators such as the Colpitts oscillator used in conjunction with a quartz resonator.

Quartz oscillators show a well-known phase (and hence frequency) fluctuation behavior observed to follow a series of power law functions. The power spectral density of relative frequency fluctuations is thus $S_{y}(F)=h_{\alpha} F^{\alpha}$, where $F$ is the Fourier frequency, $y=\Delta \nu / \nu_{0}$ the relative frequency shift with respect to the carrier of mean frequency $\nu_{0}$ and the integer $\alpha$ varies from -2 to +2 . The power spectral density of phase fluctuations is $S_{\varphi}(F)=\left(\nu_{0} / F^{2}\right) S_{y}(F)$. Hence a slope equal to -3 in $S_{\varphi}(F)$ corresponds to $1 / F$ noise in $S_{y}(F)$. This type of noise is still not well understood. This is one reason for attempting to detect it in a Chua's oscillator. Instead of power spectra we will use the characterization of time dynamics in terms of Allan variance which is defined as $\sigma_{y}^{2}(\tau)=(1 / 2)\left\langle\left(y_{k+1}(\tau)-y_{k}(\tau)\right)^{2}\right\rangle$, with $y_{k}(\tau)$ the $k$ th average of samples $y$ over the integration time $\tau$. The coefficient $y$ is obtained by computing 

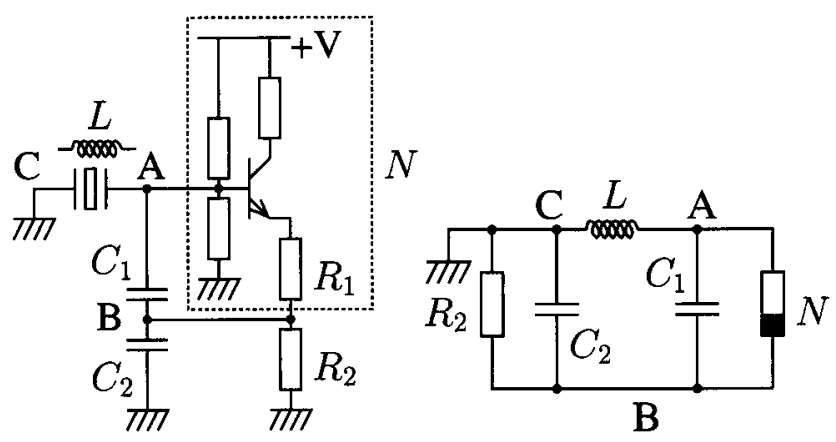

Fig. 1. Comparison of the Colpitts oscillator often used for using a quartz crystal resonator in an oscillator configuration, and the canonical Chua circuit.

the average $y_{\tau}=\left\langle x_{n=[t ; t+\tau]}\right\rangle, x_{t}$ being our time series indexed by $t$ and $\langle\bullet\rangle$ an average over the data sets. For a stationary fluctuation the power spectrum and Allan variance are related. The graph of the variance against the sampling time also obeys power laws $\sigma_{\tau}^{2}(y)=\tau^{-m}$, with $m=\alpha+1$ (if $-2 \leq \alpha \leq 1$ ) and $m=2$ (if $\alpha \geq 1$ ). Therefore the $1 / F$ noise in $S_{y}(F)$ corresponds to a flicker floor $m=0$. Allan variance is often used for describing the stability of oscillators since it converges for the kinds of noises observed experimentally, as opposed to the classical variance which does not always converge to a finite value [Allan et al., 1997; Audoin et al., 2001].
A parallel between the schematics of the Colpitts oscillator and the canonical Chua's circuit [Sarafian \& Kaplan, 1995; Kennedy, 1995] can be displayed and analyzed in order to justify our extension of the results to quartz oscillators (Fig. 1). The circuit we actually studied is the usual Chua's circuit in which $L$ and $R_{2}$ are exchanged [Chua \& Lin, 1990] as shown in Fig. 3. The nonlinear differential equations used to predict the behavior of the circuit lead to chaotic oscillations after a series of bifurcations. Here we have studied simultaneously the evolution of the instantaneous amplitude and instantaneous frequency with the value $L$ of the inductor parameter.

\section{Experimental Setup}

A preliminary study shows a large variation in the shape of the power spectra (Fig. 2) observed on Chua's circuit for various kinds of attractors (from periodic to chaotic behaviors of the circuit with varying values of the bifurcation parameter). We will here attempt to precisely characterize the various fluctuation regimes depending on the inductor value by means of signal processing tools including the Allan deviation and a display of return maps.

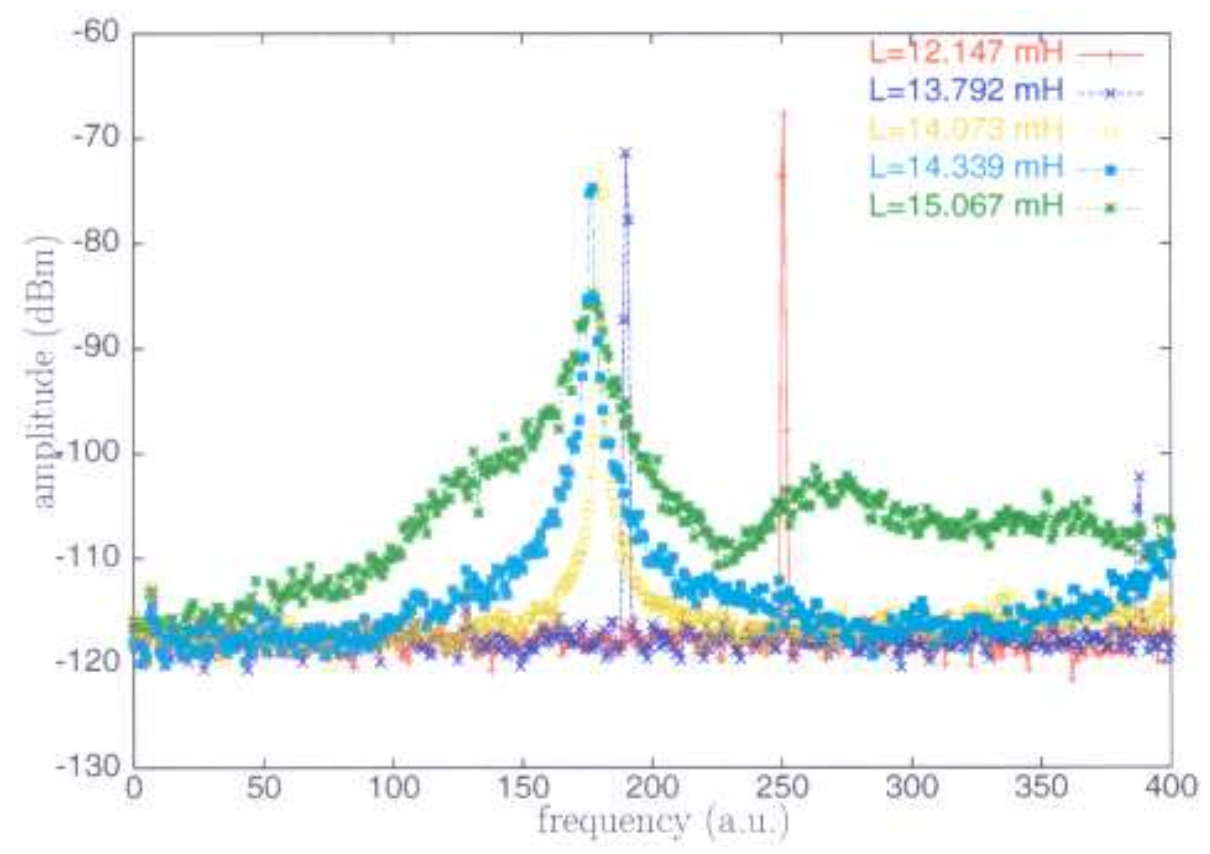

Fig. 2. Evolution of the power spectra around the oscillation frequency of Chua's circuit. A precise analysis is needed to describe the slope of the spectra depending on the oscillation regime. 


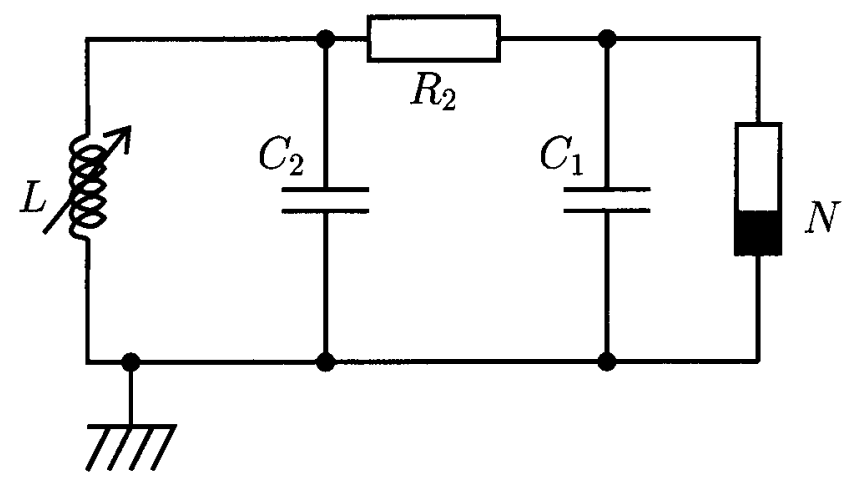

Fig. 3. Schematic of Chua's circuit we used for our data acquisitions. The parameter is the inductor's value $L$. Other components value are $C_{2}=1847 \mathrm{nF}, C_{1}=20.3 \mathrm{nF}$, inductor's internal resistance $3.3 \Omega$. $R_{2}$ is a $10 \mathrm{k} \Omega$ variable resistor. The variables measured are the frequency and amplitude of the voltage at $C_{2}$.

For the realization of Chua's circuit, we used a simulated inductor described by Weldon [1990]: it is less bulky and its value is defined by a variable resistor and thus can be computer controlled thanks to the availability of computer controlled variable resistors. The nonlinear negative resistor is a negative impedance converter (NIC) [Horowitz \& Hill, 1989] based on an operational-amplifier [Weldon, 1990]. The details of the electronic circuits we built are given in Fig. 4.

An external circuit displayed in Fig. 5 is used for detecting single periods and measuring their duration thanks to a comparator and a frequency counter with an internal clock at $14.31818 \mathrm{MHz}$. The amplitude is measured simultaneously by using a peak holder circuit and a 12 bit analog to digital converter. The trigger signal for the frequency measurement and the amplitude signal can be seen on Fig. 6. All consecutive periods can be measured for signals of frequency less than $1 \mathrm{kHz}$.

\section{Statistical Analysis and Bifurcation Diagrams}

All our statistical analysis have been limited to regions of the $L$ parameter for which the signal is observed to be periodic or pseudo-periodic with a constant mean value (as opposed to smaller oscillations overlapping large scale fluctuations such as the signals observed in the double scroll oscillator). This limitation is required by our data acquisition electronic circuit as well as by our intuitive definition of frequency and amplitude of pseudo-periodic signals.

We plotted the values taken by the amplitude and frequency time series against the value of the $L$ parameter in Figs. 7 and 8 . One can observe the first bifurcation, followed by the chaotic behavior [Feigenbaum, 1978, 1979].

We also display the Allan deviation $\sigma_{y}(\tau)$ : a -0.5 slope in the Allan deviation indicates a white frequency noise, while a -1 slope indicates $\alpha=+1$ (flicker phase fluctuations) or $\alpha=+2$ (white phase noise) frequency fluctuation spectra (Fig. 8).

We then display the evolution of various parameters which are characteristic of the dynamic of Chua's circuit for several chosen values of the $L$ parameter: the frequency return maps (Fig. 9), the transfer functions (Fig. 10) and the amplitude return maps (Fig. 11). The return map is defined by displaying $f_{n+1}$ versus $f_{n}$ when given a time series $\left(f_{n, n \in[1 . . N]}\right)$. The amplitude and frequency measured are similar to their usual definition when the

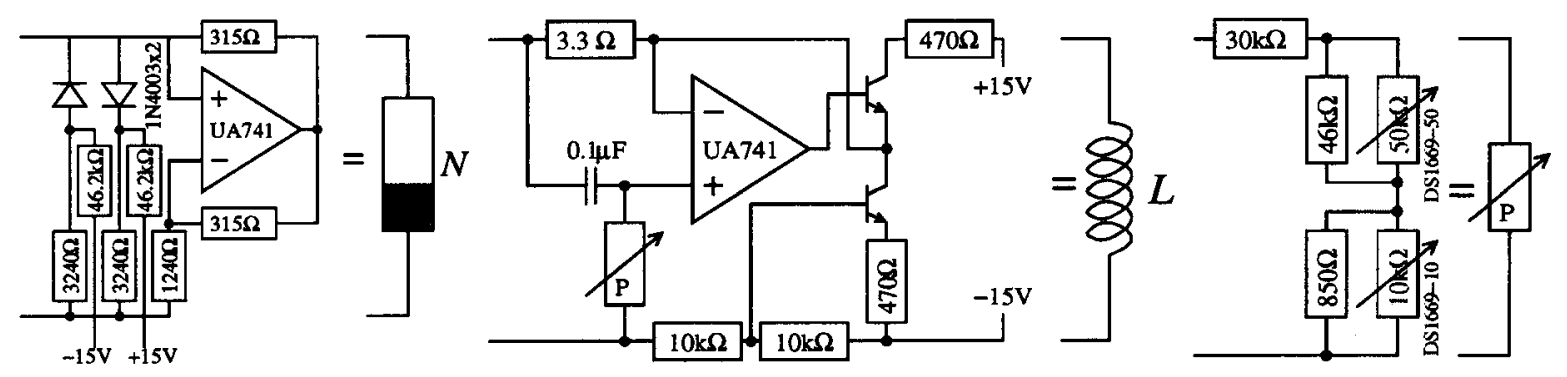

Fig. 4. Details of the electronic circuits used in these experiments. The nonlinear element is described in the left schematic, the simulated inductance is depicted in the middle schematic and includes a computer controlled variable resistance, $P$, whose detailed schematic is presented on the right. The DS1669 computer controlled linear potentiometers are powered between $-1.44 \mathrm{~V}$ and $5.28 \mathrm{~V}$. The value of the simulated inductance is $L=0.1 \times 10^{-6} \times 3.3 \times P \mathrm{H}$. 


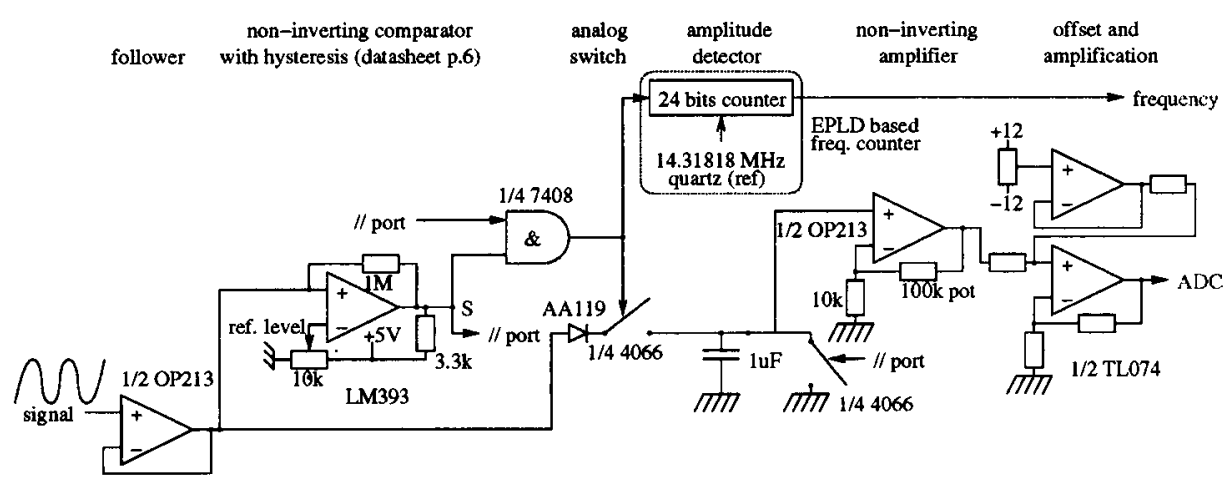

Fig. 5. Experimental setup used for measuring the frequency and amplitude of successive oscillations of Chua's circuit. A buffer was included so as to minimize the disturbances on the chaotic circuit due to the measurement apparatus. The diode used for the amplitude detection is a germanium diode in order to minimize the voltage drop. The frequency counter and the analog to digital converter (12 bits) are custom built PC-104 cards: this allows fast transfer time and precise control of the timings, allowing all successive periods to be measured during a given data acquisition time lapse.
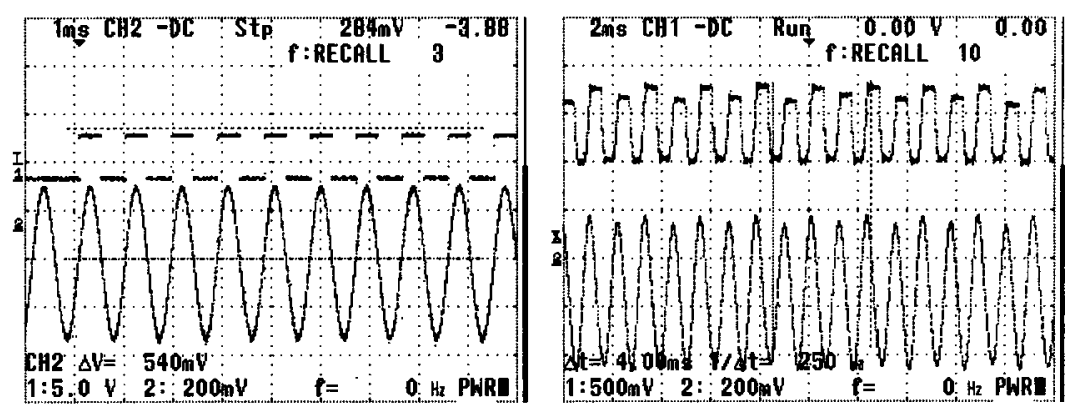

Fig. 6. Trigger signal for the frequency measurement (left, top curve) and amplitude measurement analog signal (right, top curve) output from the electronic circuit connected to Chua's circuit. In both images, the bottom curve is the signal observed at the output of Chua's circuit (chaotic regime). The amplitude signal (right) is read by a 12 bit analog to digital converter while a custom made frequency counter samples the pulse (left). Both values are read in less than $500 \mu \mathrm{s}$, half of the duration of a period of Chua's circuit, and allows the measurement of all successive periods.

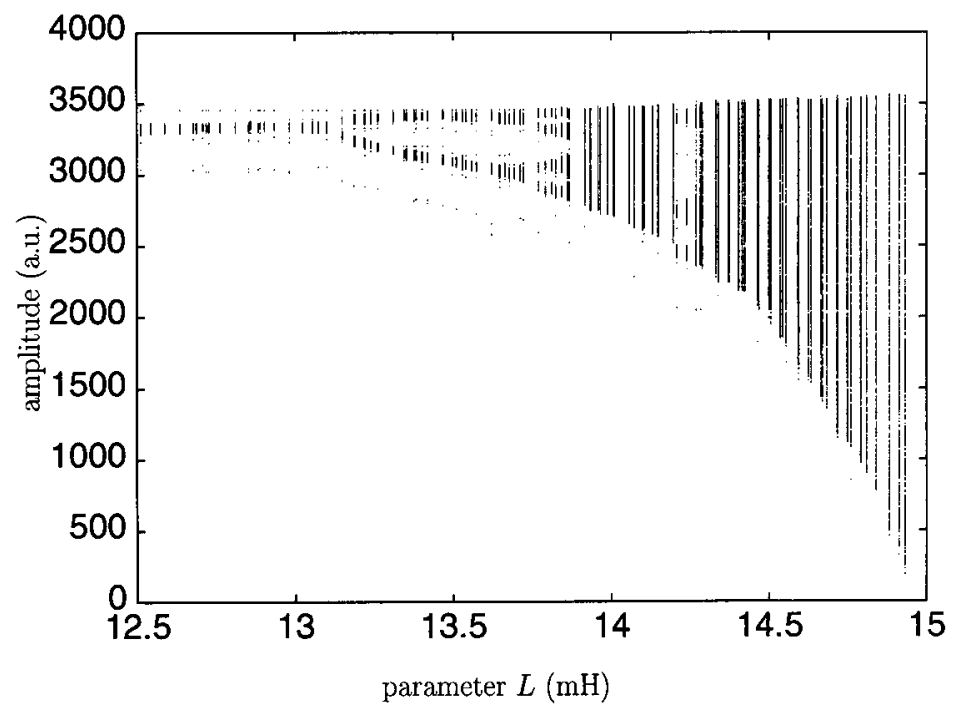

Fig. 7. Bifurcation diagram for the amplitude data. 

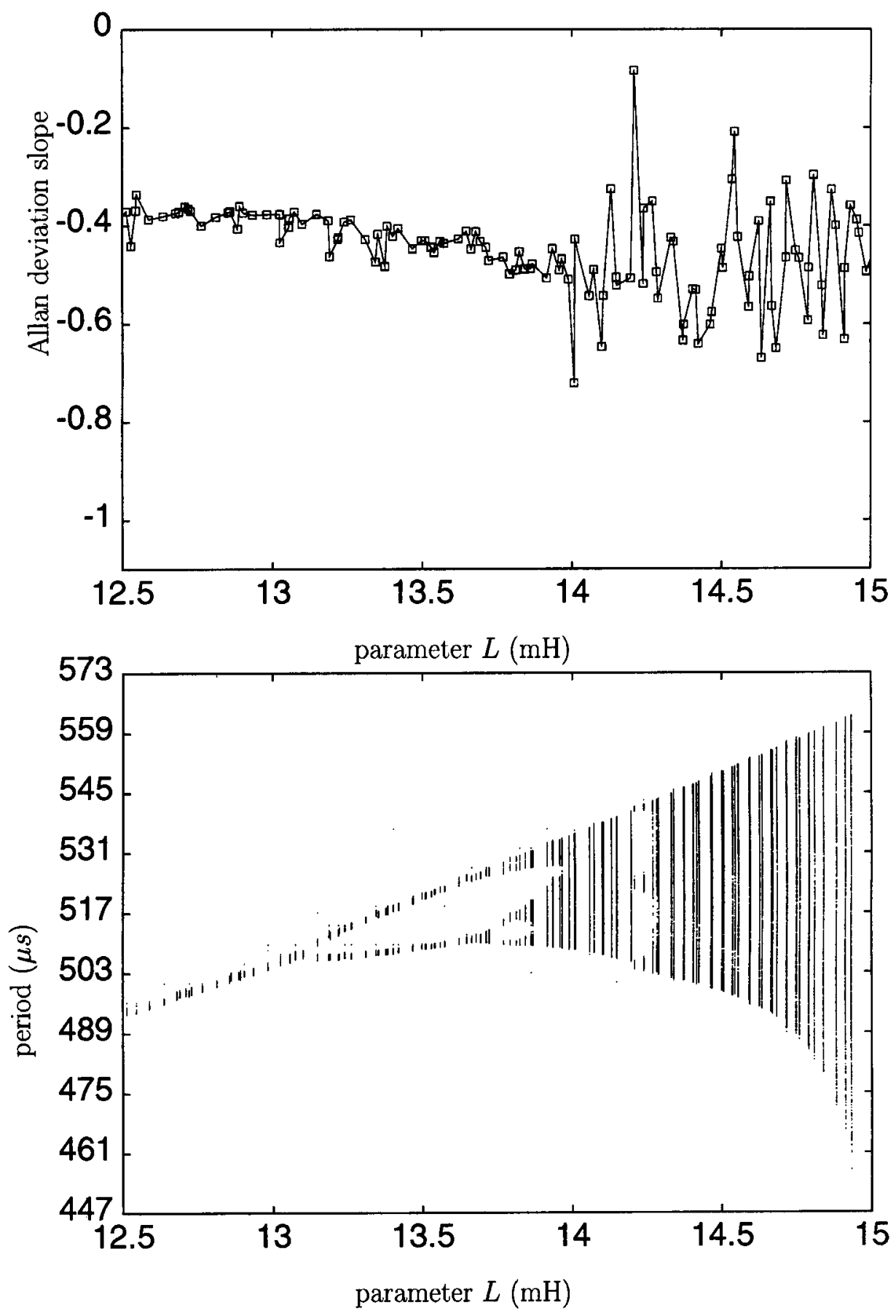

Fig. 8. Slope of Allan deviation (top) and bifurcation diagram (bottom) for the frequency data.

signal is periodic, and is intuitively extended to respectively the maximum of the voltage over each period and the inverse of the duration between two crossings of a reference level, chosen here to be $0 \mathrm{~V}$ (ground level being the average value of the voltages in the periodic and quasi-periodic regimes). Figure 6 illustrates these definitions by displaying both the signal being analyzed (bottom signal) and the frequency (left) and amplitude (right) measurements (top waveforms). We have selected values of the parameter for which we can see a periodic signal followed by signals characteristic of each further bifurcation, until obtaining a fully developed (Rössler like) chaotic attractor. The chosen values of $L$ are the following: $13.167 \mathrm{mH}$ for a periodic signal, $13.497 \mathrm{mH}$ and $13.926 \mathrm{mH}$ for signals after 


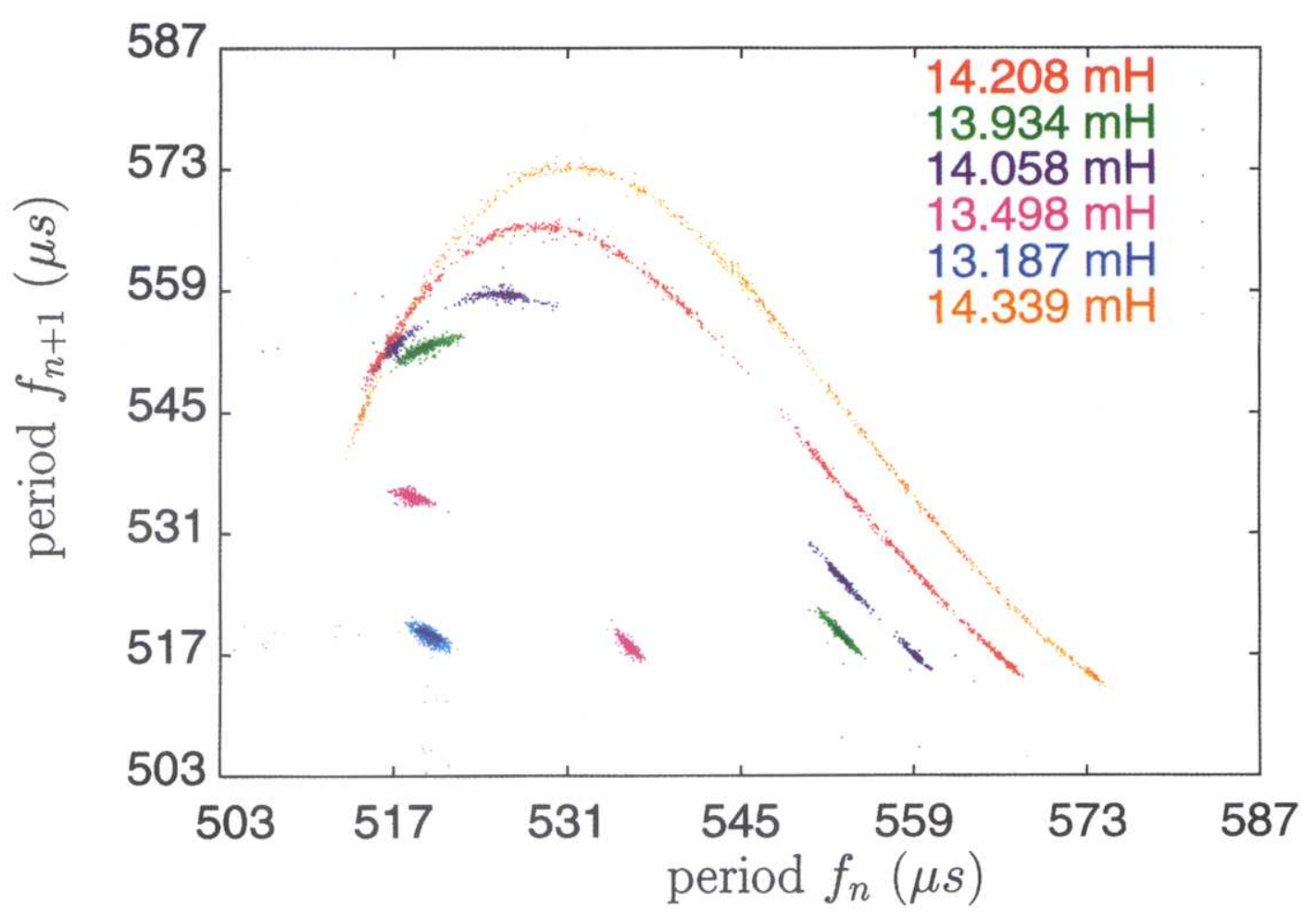

Fig. 9. Evolution of the return maps $\left(a_{n}\right.$ versus $\left.f_{n}\right)$ for the period data with the $L$ parameter.

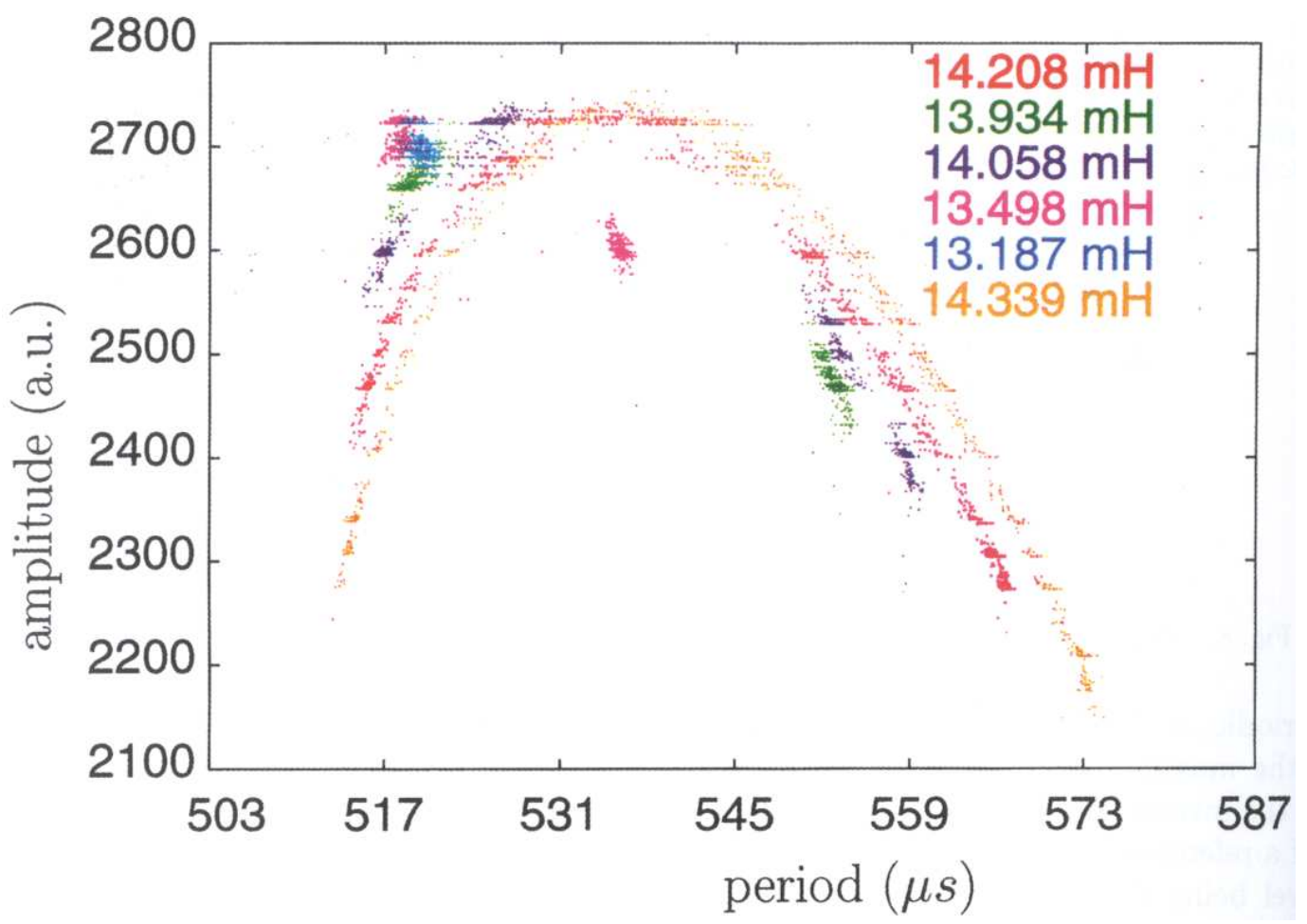

Fig. 10. Evolution of the transfer functions (the abscissa are not here the frequency as usually used when displaying transfer functions, but the period, which is more relevant in this study) with the parameter $L$. 


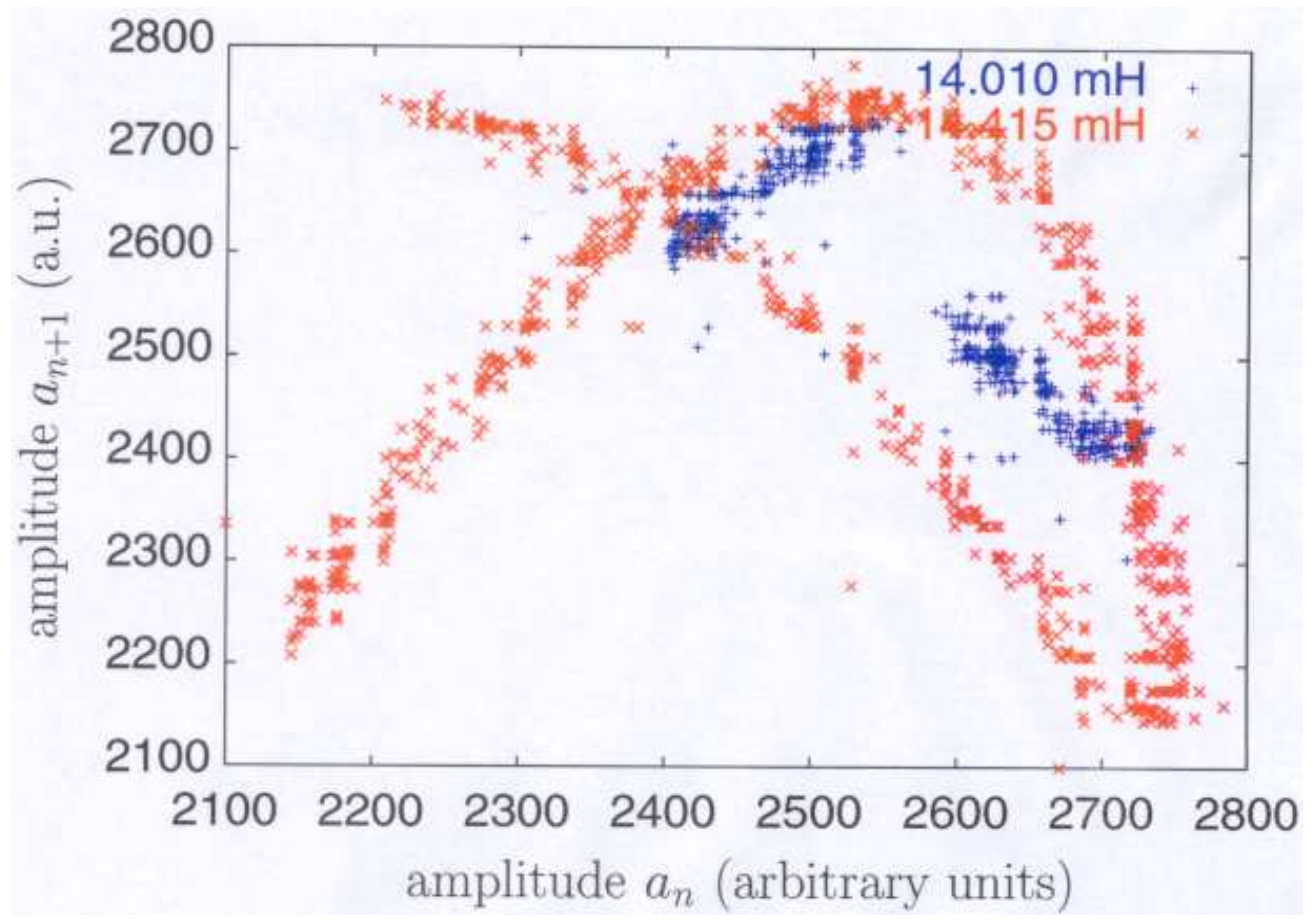

Fig. 11. Evolution of the return maps for the amplitude data with the $L$ parameter. Notice that the relation between $a_{n}$ and $a_{n+1}\left(a_{n}\right.$ being the amplitude data) is no longer a function for a value large enough of $L$.

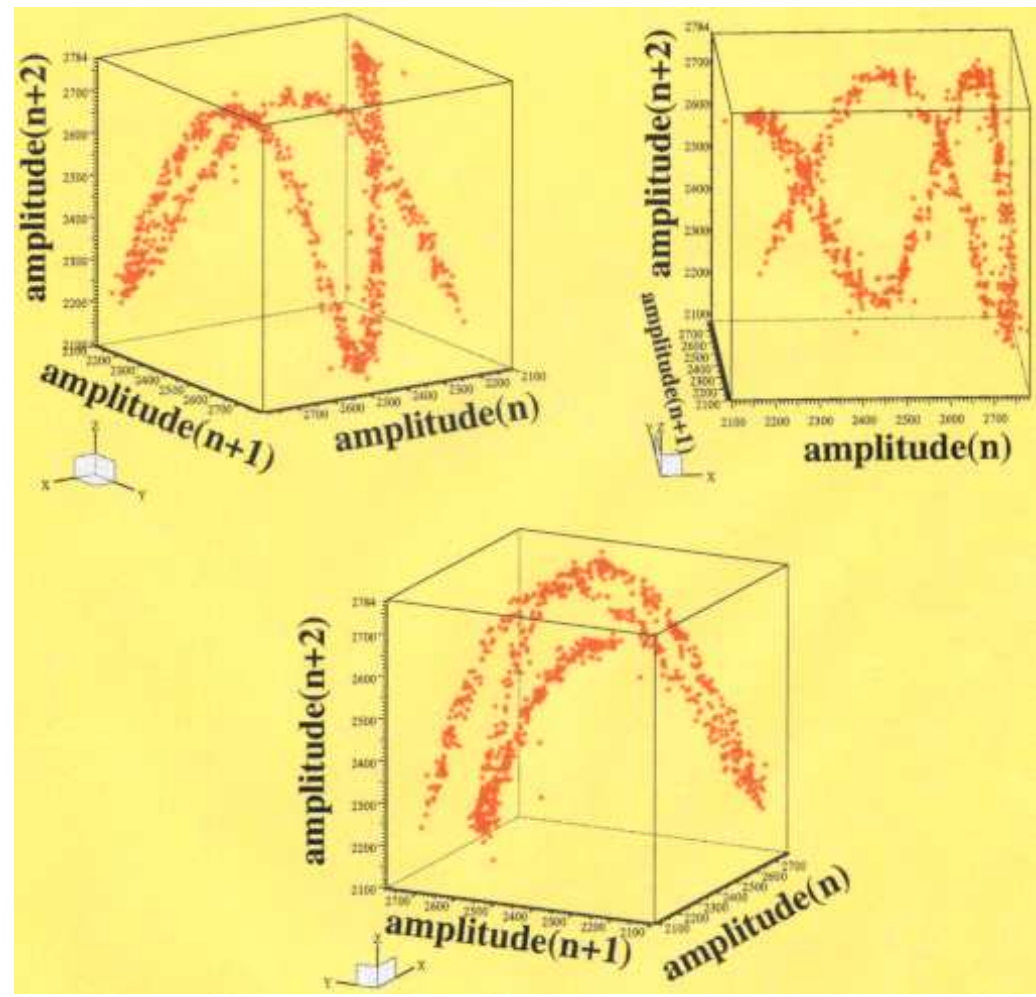

Fig. 12. 3D plots of the experimental amplitude return map at 1 and 2 steps in the chaotic regime of the circuit. $L=$ $14.415 \mathrm{mH}$. These plots can be compared with Fig. 11: adding a third dimension unwraps the return map. 
the first bifurcation, $14.207 \mathrm{mH}$ for a signal after the second bifurcation, and finally $14.058 \mathrm{mH}$ and $14.322 \mathrm{mH}$ for fully developed chaos (Rössler-like attractor).

Figure 10 displays the evolution of the transfer functions with the parameter $L$. Absicissa are here given in duration units, rather than in the usual frequency units as classically used. The splitting of the fundamental spot (top-left) is clearly identified. A major difference between the return maps of the frequency data (Fig. 9) and the return maps of the amplitude data (Fig. 11) can be observed: the return maps of the frequency data are always functions ( $f_{n}$ defines one $f_{n+1}$ only) while return maps of the amplitude data are no longer functions for large enough values of the parameter $L$. By adding additional dimensions, i.e. plotting $a_{n}$ as a function of $a_{n+1}$ and $a_{n+2}\left(a_{n, n \in[1 . . N]}\right.$ being the amplitude data), the relation becomes a function again by unwrapping the return map, and the prediction is again possible (Fig. 12).

\section{Conclusion}

After validating our data acquisition electronics and software by plotting the bifurcation diagrams, we have shown the experimental return maps and transfer functions of Chua's circuit. We have then analyzed the frequency fluctuations using the Allan deviation.

The slope of the Allan deviation was shown to be equal to -0.5 over most of the bifurcation parameter range, meaning the frequency fluctuations are mainly characterized by white frequency noise. The same kind of behavior is observed in quartz crystal based oscillators far enough from the carrier (100 kHz to $1 \mathrm{MHz}$ from the $11.0592 \mathrm{MHz}$ carrier for a Colpitts oscillator).

\section{References}

Allan, D. W., Ashby, N. \& Hodge, C. C. [1997] "The science of timekeeping," Hewlett Packard Application Note 1289.

Audoin, C., Guinot, B. \& Lyle, S. [2001] The Measurement of Time (Cambridge University Press).

Chua, L. O. \& Lin, G.-N. [1990] "Canonical realization of Chua's circuit family," IEEE Trans. Circuits Syst. 37, 885-902.

Eckert, C., Miehe, J. \& Planat, M. [1996] "On the hidden order in the frequency noise of an electronic oscillator," Phys. Rev. 54, 6093.

Feigenbaum, M. [1978] "Quantitative universality for a class of nonlinear transformations," J. Statist. Phys. 19, 158-185.

Feigenbaum, M. [1979] "The universal metric properties of nonlinear transformations," J. Statist. Phys. 21, 669-706.

Horowitz, P. \& Hill, W. [1989] The Art of Electronics (Cambridge University Press).

Kennedy, M. P. [1995] "On the relationship between the chaotic colpitts oscillator and Chua's oscillator," IEEE Trans. Circuits Syst. 42, 376-379.

Sarafian, G. \& Kaplan, B. Z. [1995] "Is the Colpitts a relative of Chua's circuit?" IEEE Trans. Circuits Syst. 42, 373-376.

Weldon, T. P. [1990] "An inductorless double scroll chaotic circuit," Am. J. Phys. 10, 936-941. 